\title{
Child and Maternal Correlates of Picky Eating in Young Children
}

\author{
Ada H. Zohar ${ }^{1,2}{ }^{*}$, Lilac Lev-Ari1,2, Rachel Bachner-Melman' ${ }^{1}$ \\ ${ }^{1}$ Graduate Program in Clinical Psychology, Ruppin Academic Center, Emek Hefer, Israel \\ ${ }^{2}$ The Lior Tsfaty Center for Suicide and Mental Pain Studies, Emek Hefer, Israel \\ Email: ^adaz@ruppin.ac.il
}

How to cite this paper: Zohar, A. H. Lev-Ari, L., \& Bachner-Melman, R. (2019). Child and Maternal Correlates of Picky Eating in Young Children. Psychology, 10, 1249-1261.

https://doi.org/10.4236/psych.2019.109080

Received: January 27, 2019

Accepted: July 6, 2019

Published: July 9, 2019

Copyright (c) 2019 by author(s) and Scientific Research Publishing Inc. This work is licensed under the Creative Commons Attribution International License (CC BY 4.0).

http://creativecommons.org/licenses/by/4.0/

(c) (i) Open Access

\begin{abstract}
Background: Picky eating (PE) is common in childhood but if it persists may pose a serious risk factor for restricting eating disorders. Methods: A community sample of 1055 mothers of children (mean age $3.4+1$ ) was reported online on gestational history, early feeding [breastfeeding vs. formula], behavioral problems $[\mathrm{CBCL}]$, temperament [EAS], fears [FIYC], executive function [P-BRIEF], and self-reported on maternal perfectionism, anxiety, and on maternal attachment patterns. Results: $17.5 \%$ of the children were PE with an over-representation of first-borns (Chi-square $=28.1, p<0.001$ ). In temperament, PEs were shyer, more emotional and fearful than non-PEs, and higher on the CBCL-PDD, Affective, Anxiety, ADHD, and Oppositional, and lower in executive function. PEs' mothers were more perfectionistic and anxious and scored higher on avoidant and anxious attachment. Logistic regression identified $26.7 \%$ of the PEs with the CBCL Affective providing most of the explained variance. Conclusions: At the age of three, $\mathrm{PE}$ is associated with a wide range of behavioral problems and with poorly developed executive function. Maternal anxiety and perfectionism may contribute to PE at this developmental stage. Reassuring anxious and perfectionistic mothers may contribute to dealing effectively with PE.
\end{abstract}

\section{Keywords}

Picky Eating, Children, Behavior Problems, Feeding, Executive Function, Maternal Perfectionism, Maternal Anxious Attachment, Maternal Trait Anxiety

\section{Introduction}

Presented here is a large-scaled community-based correlational study of picky eating in young children, measuring a wide range of child and maternal charac- 
teristics and behaviors, so as to best describe those children who are picky eaters as well as their mothers, in contrast to age-matched children who are not picky eaters.

Picky eating has been defined in various ways, but most definitions include a highly restrictive diet and a neophobia, a revulsion of new foods. A series of twin studies (Knaapila et al., 2007; Wardle \& Cooke, 2008; Faith, Heo, Keller, \& Pietrobelli, 2013) supported high heritability for neophobia, ranging from $60 \%$ to $80 \%$. Cooke Haworth and Wardle (2007) found that most of the remaining variance could be attributed to non-shared environment.

Feeding and eating are a central feature of the parent-child relationship and of the home culture (Cole, Musaad, Lee, Donovan, \& The STRONG Kids Team, 2018; Faith, Scanlon, Birch, Francis, \& Sherry, 2004), and multiple studies have shown a relationship between parental feeding practices and children's eating behavior and BMI, from childhood through to adulthood (e.g. Lev-Ari \& Zohar, 2013). Picky eating in childhood is associated with poor health. Chao (2018), reported that the picky eaters in his sample of toddlers were thinner, and smaller, were less physical active, and more prone to constipation than non-picky eaters, and were twice as likely to be sick in the study time-frame. Consistently and extremely restrictive eaters are at risk for a restrictive eating or feeding disorder such as avoidant/restrictive food intake disorder (ARFID; Fisher et al., 2014). In the long-term, children who are picky eaters at the age of three tend to be heavier than average as young adults and are at higher risk for obesity than adults who were not picky eaters as children (Taylor, Steer, Hays, \& Emmet, 2018).

Cano et al. (2015) studied nearly 4000 children from the age of 18 months, assessing them every 18 months until they were seven. Four different trajectories could be discerned: Children who were never picky eaters (51.4\%); those who went through an early, transient phase of pickiness $(31.9 \%)$; those who were consistently picky at each time point (5.5\%) and those who were first described as picky at six years of age ("late onset", $4.7 \%$ ). An additional $6.5 \%$ were inconsistent and could not be classified into one of the four trajectories. Cano et al. (2016) found that the children with transient and "late-onset" picky eating were not at increased risk for emotional or behavioral problems and concluded that a phase of picky eating in young children is developmentally normal. However, the early and persistent picky eaters were at increased risk of attention/hyperactivity behavioral problems, of oppositional behavior, and their risk for pervasive developmental delayed behavior problems was double that of children who were never picky eaters.

The goal of the current study was to assess a wide range of potential child and mother correlates of picky eating. The children at the time of ascertainment were all in preschool, i.e. in daycare centers for children under the age of five. These preschool children were studied so as to deepen our understanding of picky eating at this age, when it is relatively common, and in order to inform pediatric healthcare professionals of best practice. In particular, we wished to examine the association between child temperament, fears, behavioral problems, and execu- 
tive function with early picky eating, as well as maternal traits that might contribute to stress in the feeding and eating dynamics of mother and child-anxiety, perfectionism and insecure attachment.

\section{Methods}

\subsection{Participants}

Participants were 1055 Hebrew-speaking volunteers from the community, mothers of children $2-6$ years old (mean $=3.4$ years, $S D=1$ ). The mothers had a mean of 2.44 children, with a range of $1-12$ children. Each mother reported about one child only. Nearly all the mothers were married (93.4\%); the remainder in order of frequency were cohabiting, divorced, single, or widowed. Of the children, $52.1 \%$ were male. Although over half of the children were firstborn (55.2\%), many were lower down in the birth order, including 2 who were 10th. The mean birth order of the child for the entire sample was 1.8 . Most $(82.6 \%)$ of the mothers reported an uncomplicated pregnancy and delivery. Of the remainder, complications of pregnancy mentioned included fertility treatments such as IVF, pregnancy diabetes, pre-eclampsia toxemia, hyper-coagulation, premature contractions, hypertension, and infections. Delivery complications reported were cesarean section, vacuum extraction, premature birth, multiple births, and fetal distress. To get a representative sample of Israeli preschool children a special effort was made to ascertain children who are raised in closed, strict, religious, Jewish communities that do not usually participate in research the "ultraorthodox". Mothers in this community were ascertained through trusted community key-members, and since they are not internet-connected these mothers reported on a paper form, anonymously. This is the fastest growing sector of Israeli society, and the most understudied (Malach, Choshen, \& Cahaner, 2016).

\subsection{Procedure}

The study was approved by the Institutional Review Board and participants provided signed informed consent. Most participants reported online via Qualtrics (2005), but a paper and pencil version was used for the ultra-orthodox participants who were not internet-connected. Pre-school daycare centers strategically chosen to provide a representative sample of the population formed the nodes for soliciting participants. Since ultraorthodox Jews are typically very opposed to participation in research, special measures were taken to include them through trusted key members of the community. All respondents were rewarded with an age- gender- and culture-appropriate children's book. All data was downloaded from Qualtrics (2005) into SPSS 23 for Windows.

\subsection{Instruments}

\section{Mother-on-Child Report:}

1) The Emotion Activity and Sociability (EAS) was used to assess child tem- 
perament. The EAS is a parental report composed by Buss and Plomin (1984) and includes 20 items that load onto four temperament sub-scales: negative emotionality (E), level of activity (A), level of sociability (S) and shyness (SH). The EAS performs well in different languages and cultures. In the current study, the lower bound reliability estimates of the temperament subscales were: E: $\alpha=$ 0.84 ; A: $\alpha=0.70$; S: $\alpha=0.71$; SH: $\alpha=0.65$.

2) The Child Behavior Checklist Pre-School (CBCL) (Auerbach, Yirmia, \& Kamel, 1996) is widely used to assess behavioral problems and has age-appropriate versions for each age group. In the current study the overall lower bound reliability estimate of the CBCL was $\alpha=0.92$; for the affective subscale it was $\alpha=$ 0.53 ; for the anxiety subscale it was $\alpha=0.65$; for the PDD subscale it was $\alpha=$ 0.67 ; for the ADHD subscale it was $\alpha=0.75$; and for the oppositional behavior subscale it was $\alpha=0.78$. For the internalizing subscale the overall lower bound reliability estimate was $\alpha=0.75$; and $\alpha=0.84$ for the externalizing subscale. The CBCL also includes 12 items of free text report, on specific behavioral problems.

3) Picky eating was defined using the relevant item of the CBCL following the Cano et al., 2015 method. In addition, a free text item of the CBCL about eating problems was analyzed, so that the content perceived by the mothers could be used to better describe the picky eating.

4) The Behavior Rating Inventory of Executive Function Preschool version (BRIEF-P; Gioia, Espy, \& Isquith, 2002) assesses executive function on preschool children. Two subscales of the BRIEF-P were used, both components of behavior regulation: shifting and emotional control. In the current study, the shifting subscale had a lower bound reliability of $\alpha=0.89$ and the emotional control had a lower bound reliability estimate of $\alpha=0.92$. Higher scores on BRIEF-P subscales denote more problematic or less developed executive function.

5) The Fear Inventory for Young Children (FIYC; Zohar \& Felz, 2001) includes 23 items that list common childhood fears and are scored on a Likert-type scale scored between 1 (not afraid at all) and 4 (very afraid). In the current study the FIYC had a lower bound reliability estimate of $\alpha=0.82$. The FIYC has four subscales: fear of imaginary figures and stories (Monsters) which had a lower bound reliability estimate of $\alpha=0.69$; Fear of strangers and novel situations (Strangers) which had a lower bound reliability estimate of $\alpha=0.68$; Fear of harm and death (Harm) which had a lower bound reliability estimate of $\alpha=$ 0.68 ; and fear of dark and night terrors (Night) which had a lower bound reliability estimate of $\alpha=0.52$.

\section{Mother Self-Report.}

6) The Frost Multidimensional Perfectionism Scale (FMPS; Frost, Lahart, \& Rosenblate, 1991) is a 35-item multidimensional self-report scale of perfectionism containing six subscales: Concern over Mistakes, Personal Standards, Parental Expectations, Parental Criticism, Doubts about actions and Organization. The FMPS has good reliability and construct validity (Frost et al., 1991). Responses are scored on a 5-point scale ranging from strongly disagree (1) to strongly agree (5). The lower bound reliability estimate of the FPMS in the cur- 
rent study was $\alpha=0.86$.

7) The Experiences in Close Relationships questionnaire (ECR; Fraley, Heffernan, Vicary, \& Brumbaugh, 2011) assesses two constructs: anxious and avoidant attachment, each measured by 6 items. The reliability of the anxious attachment scale in this study was $\alpha=0.79$ and of the avoidant scale $\alpha=0.63$.

8) The Spielberger Trait Anxiety Inventory (STAI; Spielberger \& Sydeman, 1994) measures trait anxiety and includes 20 items that are scored on a Likert-type five-point scale. In the current study trait anxiety had a lower bound reliability estimate of $\alpha=0.90$.

\section{Data Analysis:}

Data analysis was conducted in SPSS for Windows version 23 and included descriptive statistics, analysis of variance, and binary logistic regression.

\section{Results}

\subsection{Description of Picky Eating}

On the free-text item of the CBCL that asks about problems with eating, most of parents, who reported picky eating, 69\%, also wrote a comment. These comments were read and analyzed. The themes most frequently mentioned were pickiness, fear of new foods, avoidance of certain textures, and a restricted range of food, in particular an avoidance of vegetables.

\subsection{Demographic Correlates of Picky Eating}

Boys and girls were equally likely to be described as picky, and 50.8\% of picky eaters were male. Social economic status was not related to the frequency of picky eating, nor was gestational history or early-feeding history. However, birth order was quite strongly associated with picky eating, with $71.6 \%$ of the picky eaters being first-born $\left(\mathrm{Chi}^{2}=28.1, p<0.0001\right)$.

\subsection{Child Psychological Correlates of Picky Eating}

Means and standard errors (SEs) of all reported child-related variables were compared for Picky vs. Non-picky eaters using MANOVA. The analysis was statistically significant $\left(\mathrm{F}_{(15,722)}=16.01 ; p<.0001\right)$, with significant group differences for most of the variables. Group comparisons and effect sizes are summarized in Table 1 and standardized means and SEs are shown in Figure 1.

Picky eaters scored higher than non-picky eaters in their fears of strangers, monsters and night terrors. Picky eaters were shyer and had higher emotionality, affective and anxiety problems, pervasive developmental disorders, ADHD and oppositional disorder than non-picky eaters, and more problematic emotional control and shifting than non-picky eaters.

Means' and SEs of all maternal self-reported variables were compared for picky vs. non-picky eaters using MANOVA. The analysis was statistically significant $\left(\mathrm{F}_{(4,721)}=4.37 ; p=.002\right)$. The two groups differed significantly on all the assessed variables, with mothers of picky eaters scoring significantly higher in 
perfectionism, trait anxiety and insecure attachment-avoidant and anxious than mothers of non-picky eaters. Group comparisons and effect sizes are summarized in Table 2 and standardized means and SEs are shown in Figure 2.

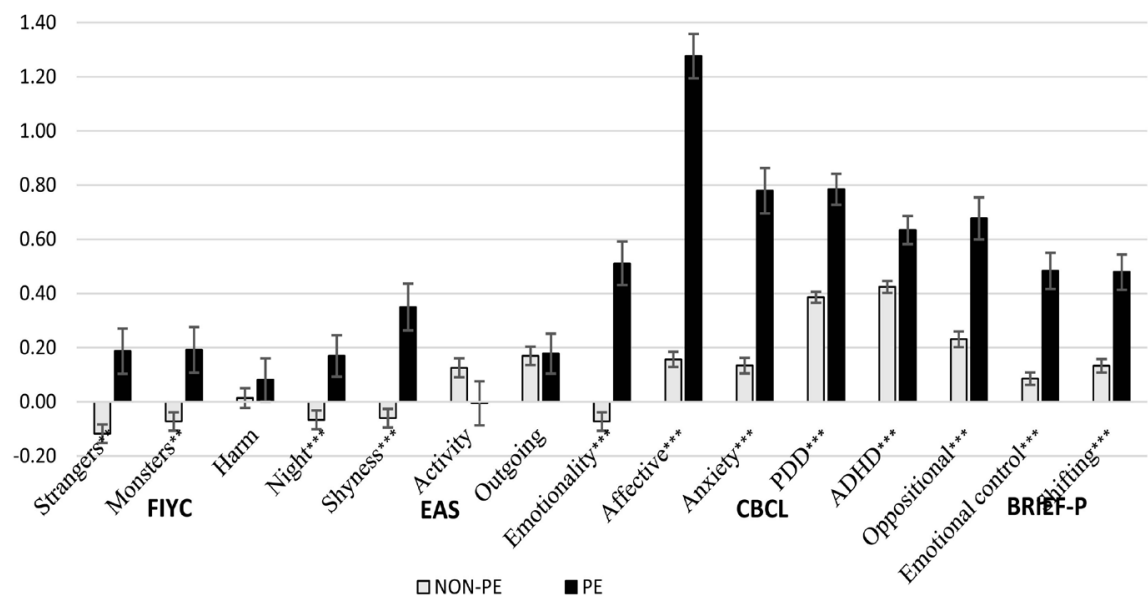

Figure 1. Standardized means and SEs of the Picky and Non-Picky Eaters on child emotional indices. Note: ${ }^{* *} p<.01 ;{ }^{* *} p<.001$. PE = Picky eaters; Non PE = Non picky-eaters; strangers, monsters, harm, night (FIYC); Shyness, Activity, Outgoing, Emotionality (EAS); Affective, Anxiety, PDD, ADHD, Oppositional (CBCL); Emotional control, Shifting (BRIEF-P).

Table 1. MANOVA results for child variables picky $(\mathrm{n}=185)$ vs. non-picky eaters $(\mathrm{n}=$ 870).

\begin{tabular}{|c|c|c|c|c|}
\hline Variable & Sub-Scale & $F_{(d f)}$ & significance & Cohen's d \\
\hline \multirow{4}{*}{ Fears (FIYC) } & Strangers & $\mathrm{F}_{(1,736)}=6.77$ & .009 & .31 \\
\hline & Monsters & $\mathrm{F}_{(1,736)}=7.19$ & .008 & .26 \\
\hline & Harm & $\mathrm{F}_{(1,736)}=.82$ & .18 & NS \\
\hline & Night & $\mathrm{F}_{(1,736)}=10.98$ & .001 & .23 \\
\hline \multirow{4}{*}{ Temperament (EAS) } & Shyness & $\mathrm{F}_{(1,736)}=12.02$ & .001 & .40 \\
\hline & Activity & $\mathrm{F}_{(1,736)}=1.16$ & .28 & NS \\
\hline & Outgoing & $\mathrm{F}_{(1,736)}=.59$ & .44 & NS \\
\hline & Emotionality & $\mathrm{F}_{(1,736)}=45.03$ & $<.0001$ & .58 \\
\hline \multirow{5}{*}{ Behavior Problems (CBCL) } & Affective & $\mathrm{F}_{(1,736)}=206.83$ & $<.0001$ & 1.12 \\
\hline & Anxiety & $\mathrm{F}_{(1,736)}=41.63$ & $<.0001$ & .65 \\
\hline & PDD & $\mathrm{F}_{(1,736)}=37.18$ & $<.0001$ & .40 \\
\hline & ADHD & $\mathrm{F}_{(1,736)}=11.68$ & .001 & .21 \\
\hline & Oppositional & $\mathrm{F}_{(1,736)}=28.59$ & .001 & .44 \\
\hline \multirow{2}{*}{ Executive Function (BRIEF-P) } & Emotional control & $\mathrm{F}_{(1,736)}=28.48$ & $<.0001$ & .40 \\
\hline & Shifting & $\mathrm{F}_{(1,736)}=15.73$ & $<.0001$ & .16 \\
\hline
\end{tabular}

FIYC $=$ Fear Inventory for Young Children; EAS $=$ Emotion Activity and Sociability; $\mathrm{CBCL}=$ Child Behavior Checklist Pre-School; BRIEF-P = Behavior Rating Inventory of Executive Function Preschool version. 


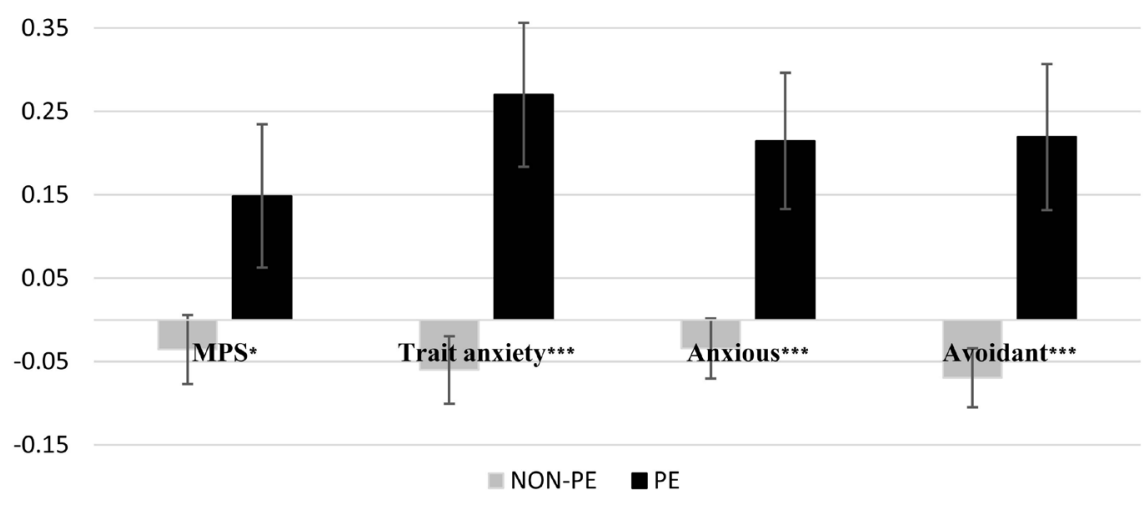

Figure 2. Standardized means and SEs of perfectionism, trait anxiety and anxious and avoidant attachment scores of the mothers of picky and non-picky eaters. Note: ${ }^{\star *} p<.01$; ${ }^{* * *} p<.001 . \mathrm{PE}=$ Picky eaters; Non-PE $=$ Non picky-eaters; MPS $=$ mother's perfectionism (FMPS); trait anxiety (STAI); anxious and avoidant (ECR).

Table 2. MANOVA results for mothers of picky $(n=185)$ vs. non-picky eaters $(n=870)$.

\begin{tabular}{cccc}
\hline & $\mathrm{F}_{(\mathrm{df})}$ & Significance & Cohen's d \\
\hline Perfectionism (FMPS) & $\mathrm{F}_{(1,736)}=3.65$ & .05 & .19 \\
Trait Anxiety (STAI) & $\mathrm{F}_{(1,736)}=11.69$ & .001 & .34 \\
Anxious Attachment (ECR) & $\mathrm{F}_{(1,736)}=8.54$ & .004 & .25 \\
Avoidant Attachment (ECR) & $\mathrm{F}_{(1,736)}=11.95$ & .001 & .29
\end{tabular}

FMPS $=$ Frost Multidimensional Perfectionism Scale; STAI = Spielberger Trait Anxiety Inventory; ECR = Experiences in Close Relationships.

\subsection{Binary Logistic Regression}

The following variables had an effect size of $d \geq .40$ in the analysis of variance and were entered into a binary logistic regression equation to predict picky vs. non-picky eaters: The EAS (temperament) scales of shyness and emotionality, the CBCL (behavior problems) sub-scales of affective problems, anxiety problems, PDD and oppositional behavior, and the BRIEF-P subscale of emotional control. None of the maternal indices had an effect size above .40, so they were not included.

The characteristics of the regression model were: Chi-square ${ }_{(7)}=150.92, p$ $<.001$; Cox \& Snell $\mathrm{R}^{2}=.184$; Nagelkerke $\mathrm{R}^{2}=.304$.

Nearly all (96.4\%) of the non-picky eaters were correctly classified. Of the picky eaters, $26.9 \%$ were correctly classified. The only significant classifier was the $\mathrm{CBCL}$ affective problems sub-scale ( $\mathrm{OR}=1.95, p<0.001)$; the greater the affective problems, the more likely the children were to be picky eaters. None of the other potential classifying variables were significant.

\section{Discussion}

This cross-sectional study of a community sample of children 2 - 6 years of age, found that $17.5 \%$ were described by their mothers as picky eaters, with an aversion to new foods and a restricted diet, in particular an avoidance of vegetables. 
Picky eating in pre-school children is quite common; Mascola, Bryson, and Agras (2010) found that between $13 \%$ and $20 \%$ of children in their study were described as picky eaters at least one time-point between the ages of 2 and 11. Cano et al. (2016) found that about a third of their community sample of children were described as picky eaters at one time or another. Children who choose to eat certain foods and not others may be asserting themselves and developing individual tastes, opposing their parents and/or seeking attention (Rubio \& Rigal, 2017) or responding emotionally to sensory aspects of foods such as taste, smell, temperature or texture (Zickgraf \& Elkins, 2018). In the majority of children picky eating is transient and will have little or no detrimental effect on development (Cano et al., 2016).

The children described as picky eaters in this study were very similar to non-picky eaters demographically. Unlike the results of Brown et al. (2018), there was no elevation of males among the picky eaters, nor was socio-economic status associated with picky eating. However, we found a substantial association with birth order; there was a significant elevation of first-born children among the picky eaters. We have found no published studies that examined the relationship of picky eating to birth order. This might be an oversight or simply reflect non-report of negative findings. Though intriguing, this result requires replication. At this point, it is possible to speculate that parents have more time and energy to invest in their firstborn children, and that this focus might not always serve the parent-child-feeding and eating dynamic well; and may in turn promote picky eating in children. Moreover, it could be that parents of first-born children are less sure of themselves, less likely to assert their authority, and be more emotionally reactive to rejection or perceived rejection of food by their (as yet) only child. Such a risk pathway was described by Steinsbekk, Bonneville-Roussy, Fildes, Llewellyn, and Wichstrøm, (2017) of sensitive parents who avoid confronting their children and tend to respond with distress to their difficulties and frustrations. A similar dynamic was described in a cross-lag study of children 21 to 33 months of age by Lumeng, Miller, Appugliese, Rosenblum, and Kaciroti, (2018). In this study, the mothers of picky eaters reported more than other mothers that they pressured their children to eat and finish their food.

In our study, mothers of picky eaters were significantly more anxious, had less secure attachment, and were more perfectionistic than mothers of non-picky eaters. The mothers of picky eaters may set themselves high standards for feeding their children, and respond with more anxiety, concern and negative emotion to their children's rejection of certain foods, exacerbating the child's tendency towards food refusal. It is also possible that a tendency to anxiety and depression characterizes both picky eaters and their mothers, demonstrating a passive gene-environment correlation (Dick, 2014). Although picky eating has been extensively studied (Yee, Lwin, \& Ho, 2017; Cole, An, Lee, \& Donovan, 2017), most studies concentrate on child-related and not mother-related variables. When variables related to maternal characteristics are studied in relation to picky eating, feeding behavior (e.g. Emmett, Hays, \& Taylor, 2018) or parental 
style (e.g. van der Horst \& Sleddens, 2017) are, understandably, the variables of choice. Nevertheless, it should be noted that the association between picky eating and mother-related variables was overall weaker than the association between picky eating and child-related variables, so that child characteristics explain picky eating to a greater extent than maternal characteristics at this developmental stage. It could be that giving additional support to anxious mothers might be helpful in the short term as well as helpful to their children's eating in the middle term.

We found that picky eaters were different from non-picky eaters in many ways. In terms of temperament, picky eaters were described by their mothers as being shyer and as expressing more negative emotion than non-picky eaters. They were also described as having more behavioral problems, especially problems related to affect and anxiety. Picky eaters were more fearful than non-picky eaters of imaginary figures, of strangers and novel situations, and of harm and death. Finally, they had more problems than non-picky eaters with executive function, specifically with emotional control and shifting. These results are consistent with findings from other studies that compared picky eaters and non-picky eaters. Adamson and Morawska (2017) found that problem eaters exhibited significantly more non-food-related behavioral problems than non-problem eaters, and Zucker, Copeland, Franz, Carpenter, Keeling, Angold, and Egger, (2015) found high levels of affective and anxiety problems, and frequent failures of self-control in selective eaters in a similar age range to that of the current study.

Most of the children in this study who were identified at a single time-point as picky eaters are probably mostly going through a phase and will expand the variety of their diet and their willingness to try new foods as they mature. This is a reasonable conjecture, since in the Cano et al. (2016) longitudinal study nearly a third of the children were identified as picky eaters at around three years of age, but only $5.5 \%$ of children remained consistently picky. It is nevertheless interesting and noteworthy that transient, "normative" picky eaters in fact exhibit a wide range of emotional and behavioral difficulties. It should, however, be pointed out that although the differences between picky and non-picky eaters were statistically significant, most had weak to moderate effect sizes. Moreover, many of the variables measured are correlated and therefore may tap into a common dimension. In a binary logistic regression using the variables that best distinguished between the groups, only $26.9 \%$ of the picky eaters were correctly identified, and only the CBCL affective problems significantly contributed to this classification function. Thus the majority of the variance of picky eating is still unaccounted for. It would be interesting to follow up the picky eaters identified in this study and to examine whether or not those correctly classified by the logistic regression model turn out to be those at high risk for persistent picky eating and future emotional and behavioral problems.

This study has several limitations. It is cross-sectional by design, and describes only one point in time, so it cannot, by definition, contribute to the understand- 
ing of causal pathways. Another central limitation is its complete reliance on maternal report. As Walton, Kuczynski, Haycraft, Breen, and Haines (2017) point out, picky eating is the parental conceptualization of the child's eating behavior, whereas a more child-centered description might reveal eating preferences and behaviors that differ from those reported by their parents. Walton et al. (2017) describe feeding and eating as a relational drama, in which both parents and children play active roles. Moreover, it is a drama that unfolds over time, and the relative roles of parents and children might change over development.

\section{Summary}

The goal of this study was to explore the behavioral pattern of picky eating and its child- and mother-related correlates in preschool children. A community sample of 1055 mothers of children (mean age $3.4 \pm 1$ ) completed a battery of online measures. Some of these measures were child-related, including gestational history, early breastfeeding vs. formula, behavioral problems (CBCL), temperament (EAS), fears (FIYC), and executive function-emotional control and shifting (BRIEF). Other measures were self-reported by the mothers, including perfectionism (MPS), anxious and avoidant attachment (ECR) and trait anxiety (STAI). $17.5 \%$ of the children were described as picky eaters and consumed a highly restricted range of foods, and had an aversion to certain food textures, and an unwillingness to try new foods. There was an over-representation of first-born children among the picky eaters. Mothers described picky eaters as being more shy, emotionally negative and fearful in temperament than non-picky eaters. They rated picky eaters higher on the CBCL subscales of PDD, Affective problems, Anxiety, ADHD and Oppositional behavior than non-picky eaters and more problematic on the executive function subscales of emotional control and shifting. The mothers of picky eaters rated themselves as more perfectionistic and anxious than other mothers and scored significantly higher on avoidant and anxious attachment. Logistic binary regression identified $26.7 \%$ of the picky eaters, with the CBCL affective problems score providing most of the explained variance. Most of the children in this study who were identified around the age of three as picky eaters are probably going through a phase and will expand the variety of their diet and their willingness to try new foods as they mature. Nevertheless, early picky eating seems to be associated with a wide range of behavioral problems and poorly developed executive function. Maternal anxiety and perfectionism may contribute to picky eating at this developmental stage.

Our results lead to the conclusion that parents should avoid power struggles overfeeding; it is unhelpful to have very strict criteria of what, when, and how much a young child of two to five years of age should eat. Rather, feeding and eating should be a pleasant interaction, in which the child may choose to eat from what is currently available, and not be pressured to eat bigger portions, or to ingest foods that the child does not wish to eat. This is particularly true of first-time parents, and of perfectionistic and anxious mothers. Paediatric clini- 
cians should give reassurance and help mothers relax and enjoy the feeding dynamic. Under these conditions, the child's picky eating behavior is more likely to be a passing phase, and the child is more likely to naturally extend his diet and enjoy eating. Moreover, the picky eating will pose less of a long-term risk for restrictive eating disorders and obesity.

\section{Acknowledgements}

The authors wish to thank the many undergraduate students and the graduate students who took part in this study, and the community volunteers, who so generously participated in this study.

\section{Author's Contribution}

AHZ designed and oversaw the execution of the study, ensured the integrity and security of the data, and wrote the original draft of the manuscript; LLA oversaw the data analyses, made the figures, and contributed substantially to the writing of the manuscript; RBM contributed substantially to the conceptualization of this study, as well as to the writing and editing of the manuscript.

\section{Funding}

Provided by an internal grant to AHZ from Ruppin Academic Center, grant number 33006.

\section{Conflicts of Interest}

The authors declare no conflicts of interest regarding the publication of this paper.

\section{References}

Adamson, M., \& Morawska, A. (2017). Early Feeding, Child Behaviour and Parenting as Correlates of Problem Eating. Journal of Child and Family Studies, 26, 3167-3178. https://doi.org/10.1007/s10826-017-0800-y

Auerbach, J., Yirmia, N., \& Kamel, F. N. (1996). Behavior Problems in Jewish and Palestinian Preschool Children. Journal of Clinical and Child Psychology, 25, 398-405. https://doi.org/10.1207/s15374424jccp2504_5

Brown, C. L., Perrin, E. M., Peterson, K. E., Herb, H. E. B., Horodynski, M. A., Contreras, D., Lumeng, J. C. et al. (2018). Association of Picky Eating with Weight Status and Dietary Quality among Low-Income Preschoolers. Academic Pediatrics, 18, 334-341. https://doi.org/10.1016/j.acap.2017.08.014

Buss, A. H., \& Plomin, R. (1984). Temperament: Early Developing Personality Traits. Hillsdale, MI: Lawrence Earlbaum.

Cano, C. S., Hoek, H. W., Van Hoeken, D., de Barse, L. M., Jaddoe, V. W., Verhulst, F. C., \& Tiemeier, W. (2016). Behavioral Outcomes of Picky Eating in Childhood: A Prospective Study in the General Population. Journal of Child Psychology and Psychiatry, 57, 1239-1246. https://doi.org/10.1111/jcpp.12530

Cano, C. S., Tiemeier, H., van Hoeken, D., Tharner, A., Jaddoe, V. W., Hofman, A., Hoek, H. W. et al. (2015). Trajectories of Picky Eating during Childhood: A General Popula- 
tion Study. International Journal of Eating Disorders, 48, 570-579.

Chao, H. C. (2018). Association of Picky Eating with Growth, Nutritional Status, Development, Physical Activity, and Health in Preschool Children. Frontiers in Pediatrics, 22, 1-9. https://doi.org/10.3389/fped.2018.00022

Cole, N. C., An, R., Lee, S. Y., \& Donovan, S. M. (2017). Correlates of Picky Eating and Food Neophobia in Young Children: A Systematic Review and Meta-Analysis. Nutrition Reviews, 75, 516-532. https://doi.org/10.1093/nutrit/nux024

Cole, N. C., Musaad, S. M., Lee, S. Y., Donovan, S. M., \& The STRONG Kids Team (2018). Home Feeding Environment and Picky Eating Behavior in Preschool-Aged Children: A Prospective Analysis. Eating Behaviors, 30, 76-82. https://doi.org/10.1016/j.eatbeh.2018.06.003

Cooke, L. J., Haworth, C. M., \& Wardle, J. (2007). Genetic and Environmental Influences on Children's Food Neophobia. The American Journal of Clinical Nutrition, 86, 428-433. https://doi.org/10.1093/ajcn/86.2.428

Dick, D. M. (2014). Gene-Environment Correlation. Wiley StatsRef: Statistics Reference Online. https://doi.org/10.1002/9781118445112.stat06727

Emmett, P. M., Hays, N. P., \& Taylor, C. M. (2018). Antecedents of Picky Eating Behaviour in Young Children. Appetite, 130, 163-173. https://doi.org/10.1016/j.appet.2018.07.032

Faith, M. S., Heo, M., Keller, K. L., \& Pietrobelli, A. (2013). Child Food Neophobia Is Heritable, Associated with Less Compliant Eating, and Moderates Familial Resemblance for BMI. Obesity, 21, 1650-1655. https://doi.org/10.1002/oby.20369

Faith, M. S., Scanlon, K. S., Birch, L. L., Francis, L. A., \& Sherry, B. (2004). Parent-Child Feeding Strategies and Their Relationships to Child Eating and Weight Status. Obesity Research, 12, 1711-1722. https://doi.org/10.1038/oby.2004.212

Fisher, M. M., Rosen, D. S., Ornstein, R. M., Mammel, K. A., Katzman, D. K., Rome, E. S., Walsh, B. T. et al. (2014). Characteristics of Avoidant/Restrictive Food Intake Disorder in Children and Adolescents: A "New Disorder" in DSM-5. Journal of Adolescent Health, 55, 49-52. https://doi.org/10.1016/j.jadohealth.2013.11.013

Fraley, R. C., Heffernan, M. E., Vicary, A. M., \& Brumbaugh, C. C. (2011). The Experiences in Close Relationships-Relationship Structures Questionnaire: A Method for Assessing Orientations across Relationships. Psychological Assessment, 23, 615-625. https://doi.org/10.1037/a0022898

Frost, R. O., Lahart, C. M., \& Rosenblate, R. (1991). The Development of Perfectionism: A Study of Daughters and Their Parents. Cognitive Therapy and Research, 15, 469-489. https://doi.org/10.1007/BF01175730

Gioia, G. A., Espy, K. A., \& Isquith, P. K. (2002). Behavior Rating Inventory of Executive Function, Preschool Version (BRIEF-P). Odessa: Psychological Assessment Resources.

Knaapila, A., Tuorila, H., Silventoinen, K., Keskitalo, K., Kallela, M., Wessman, M., Perola, M. et al. (2007). Food Neophobia Shows Heritable Variation in Humans. Physiology \& Behavior, 91, 573-578. https://doi.org/10.1016/j.physbeh.2007.03.019

Lev-Ari, L., \& Zohar, A. H. (2013). Nothing Gained: An Explorative Study of the Long Term Effects of Perceived Maternal Feeding Practices on Women's and Men's Adult BMI, Body Image Dissatisfaction, and Disordered Eating. International Journal of Psychology, 48, 1201-1211. https://doi.org/10.1080/00207594.2013.779378

Lumeng, J. C., Miller, A. L., Appugliese, D., Rosenblum, K., \& Kaciroti, N. (2018). Picky Eating, Pressuring Feeding, and Growth in Toddlers. Appetite, 123, 299-305.

https://doi.org/10.1016/j.appet.2017.12.020 
Malach, G., Choshen, M., \& Cahaner, L. (2016). The Year Book of Ultra-Orthodox Society in Israel. Jerusalem: The Jerusalem Institute of Israel Studies.

Mascola, A. J., Bryson, S. W., \& Agras, W. S. (2010). Picky Eating during Childhood: A Longitudinal Study to Age 11 Years. Eating Behaviors, 11, 253-257.

https://doi.org/10.1016/j.eatbeh.2010.05.006

Qualtrics (2005). Provo, UT, USA. https://www.qualtrics.com

Rubio, B., \& Rigal, N. (2017). Parental Concerns and Attributions of Food Pickiness and Its Consequences for the Parent-Child Relationship: A Qualitative Analysis. Journal of Child Health Care, 21, 404-414. https://doi.org/10.1177/1367493517725832

Spielberger, C., \& Sydeman, S. J. (1994). State-Trait Anxiety Inventory and State-Trait Anger Expression Inventory. In M. E. Maruish (Ed.), The Use of Psychological Testing for Treatment Planning and Outcome Assessment (pp. 292-321). Hillsdale, MI: Lawrence Erlbaum Associates.

Steinsbekk, S., Bonneville-Roussy, A., Fildes, A., Llewellyn, C. H., \& Wichstrøm, L. (2017). Child and Parent Predictors of Picky Eating from Preschool to School Age. International Journal of Behavioral Nutrition and Physical Activity, 14, 1-8. https://doi.org/10.1186/s12966-017-0542-7

Taylor, C. M., Steer, C. D., Hays, N. P., \& Emmett, P. M. (2018). Growth and Body Composition in Children Who Are Picky Eaters: A Longitudinal View. European Journal of Clinical Nutrition, 73, 869-878. https://doi.org/10.1038/s41430-018-0250-7

van der Horst, K., \& Sleddens, E. F. (2017). Parenting Styles, Feeding Styles and Food-Related Parenting Practices in Relation to Toddlers' Eating Styles: A Cluster-Analytic Approach. PLoS ONE, 12, e0178149.

https://doi.org/10.1371/journal.pone.0178149

Walton, K., Kuczynski, L., Haycraft, E., Breen, A., \& Haines, J. (2017). Time to Re-Think Picky Eating? A Relational Approach to Understanding Picky Eating. International Journal of Behavioral Nutrition and Physical Activity, 14, 62. https://doi.org/10.1186/s12966-017-0520-0

Wardle, J., \& Cooke, L. (2008). Genetic and Environmental Determinants of Children's Food Preferences. British Journal of Nutrition, 99, S15-S21. https://doi.org/10.1017/S000711450889246X

Yee, A. Z., Lwin, M. O., \& Ho, S. S. (2017). The Influence of Parental Practices on Child Promotive and Preventive Food Consumption Behaviors: A Systematic Review and Meta-Analysis. International Journal of Behavioral Nutrition and Physical Activity, 14, 1-14. https://doi.org/10.1186/s12966-017-0501-3

Zickgraf, H. F., \& Elkins, A. (2018). Sensory Sensitivity Mediates the Relationship between Anxiety and Picky Eating in Children/Adolescents Ages 8-17, and in College Undergraduates: A Replication and Age-Upward Extension. Appetite, 128, 333-339. https://doi.org/10.1016/j.appet.2018.06.023

Zohar, A. H., \& Felz, L. (2001). Ritualistic Behavior in Young Children. Journal of Abnormal Child Psychology, 29, 121-128. https://doi.org/10.1023/A:1005231912747

Zucker, N., Copeland, W., Franz, L., Carpenter, K., Keeling, L., Angold, A., \& Egger, H. (2015). Psychological and psychosocial Impairment in Preschoolers with Selective Eating. Pediatrics, 137, 582-590. https://doi.org/10.1542/peds.2014-2386 